\title{
Hipertensión arterial en la mujer adulta
}

\author{
RODRIGO TAGLE V. ${ }^{1}$, MÓNICA ACEVEDO ${ }^{2}$, GLORIA VALDÉS $^{1}$
}

\section{Hypertension in women}

The present review examines the types of hypertension that women may suffer throughout life, their physiopathological characteristics and management. In early life, the currently used low-dose oral contraceptives seldom cause hypertension. Pregnancy provokes preeclampsia, its main medical complication, secondary to inadequate transformation of the spiral arteries and the subsequent multisystem endothelial damage caused by deportation of placental factors and microparticles. Hypertension in preeclampsia is an epiphenomenon which needs to be controlled at levels that reduce maternal risk without impairing placental perfusion. The hemodynamic changes of pregnancy may unmask a hypertensive phenotype, may exacerbate a chronic hypertension, or may complicate hypertension secondary to lupus, renovascular lesions, and pheochromocytoma. On the other hand a primary aldosteronism may benefit from the effect of progesterone and present as a postpartum hypertension. A hypertensive pregnancy, especially preeclampsia, represents a risk for cardiac, vascular and renal disease in later life. Menopause may mimic a pheochromocytoma, and is associated to endothelial dysfunction and salt-sensitivity. Among women, non-pharmacological treatment should be forcefully advocated, except for sodium restriction during pregnancy. The blockade of the renin-angiotensin system should be avoided in women at risk of pregnancy; betablockers could be used with precautions during pregnancy; diuretics, ACE inhibitors and angiotensin receptor antagonists should not be used during breast feeding. Collateral effects of antihypertensives, such as hyponatremia, cough and edema are more common in women. Thus, hypertension in women should be managed according to the different life stages.

(Rev Med Chile 2013; 141: 237-247).

Key words: Hypertension; Hypertension, Pregnancy-induced; Menopause.

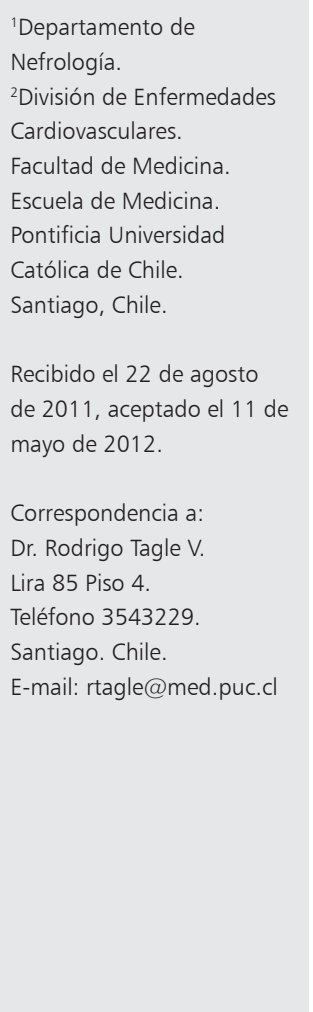

E n la mujer la hipertensión arterial (HTA) constituye una de las patologías más comunes, con una prevalencia de hasta un $80 \%$ en las adultas mayores ${ }^{1}$. Es uno de los factores de riesgo de mayor importancia para el desarrollo de cardiopatía coronaria (CC), accidente cerebrovascular $(\mathrm{ACV})^{2}$, e insuficiencia cardiaca ${ }^{3}$.

En Chile, la prevalencia de HTA en mujeres, según la Encuesta Nacional de Salud (ENS) 2003, era de $30 \%$ en mayores de 17 años $^{4}$, y en la ENS 2009 25\% en mayores de 15 años ${ }^{4}$. En ambos estudios, la prevalencia de HTA aumenta con la edad (Figura 1). La prevalencia de HTA sistólica aislada (PAS $\geq 140 \mathrm{mmHg}$ con $\mathrm{PAD}<90 \mathrm{mmHg}$ ) aumenta con la edad, en cambio la HTA diastólica aislada (PAS $<140 \mathrm{mmHg}$ y $\mathrm{PAD} \geq 90 \mathrm{mmHg}$ ) es infrecuente a mayor edad (Figura 2$)^{4}$. En la ENS 2003, las mujeres conocían más su diagnóstico que los hombres (74,7 vs $46,8 \%)$, recibían más tratamiento farmacológico (54,6 vs $20,4 \%$ ), y presentaban una proporción de HTA controlada superior ( 19,1 vs $5,4 \%$ ), aunque $35,5 \%$ no lograba normotensión bajo tratamiento. Sin embargo, el $19 \%$ bien controlado es inferior al 35\% observado en Estados Unidos ${ }^{4,5}$.

En la ENS 2009 se encontró que las mujeres con antecedente de embarazo hipertensivo tenían $25,4 \%$ de HTA crónica en el largo plazo versus $16,7 \%$ de las que presentaron embarazos normotensivos. 


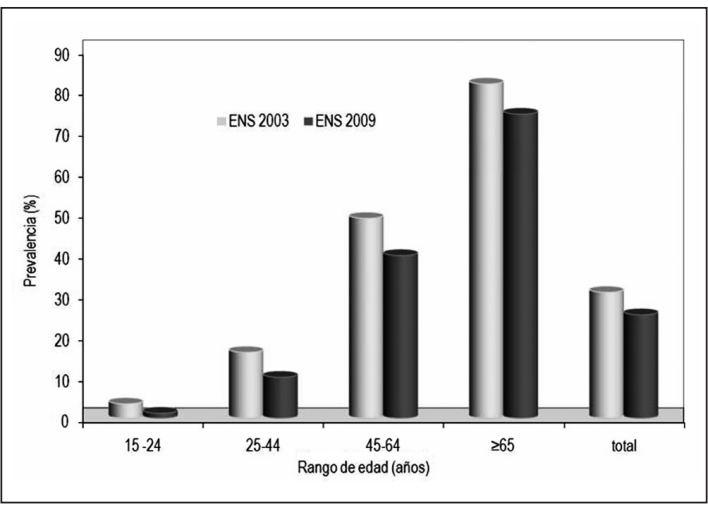

Figura 1. Prevalencia de hipertensión arterial en mujeres por edad según las Encuesta Nacional de Salud de los años 2003 y ENS 2009.

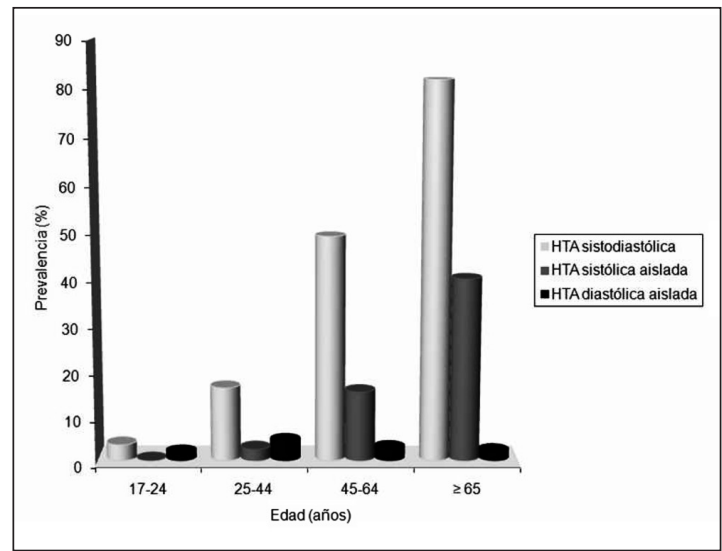

Figura 2. Prevalencia de hipertensión arterial en mujeres según tipos en la Encuesta Nacional de Salud 2003.

Además de la HTA esencial -que aparece entre los 30 a 40 años- en la mujer la HTA puede presentarse desde el inicio de la etapa fértil por uso de anticonceptivos orales (ACO), durante el embarazo y lactancia, en la postmenopausia y en la senectud, teniendo diferentes mecanismos y, en consecuencia, terapias y pronósticos distintos.

\section{Medición de la presión arterial}

La medición de la PA en la mujer se realiza siguiendo las normas de la $\mathrm{AHA}^{6}$, como en posición sentada y en el brazo con un brazalete adecuado. Sin embargo, en determinadas circunstancias deben tenerse presente ciertas consideraciones: a) En mujeres con brazo ancho y conoide, la medición de la PA es dificultosa, y tiende a sobreestimarse ${ }^{7}$. En ellas, se sugiere utilizar un brazalete grande, y si aún no se logra medir, hacerlo en el antebrazo o en la muñeca ${ }^{8,9}$.

b) En la postmenopausia, por la alta prevalencia del efecto de "delantal blanco", es recomendable agregar el registro de PA ambulatorio, ya sea MAPA o medición en el hogar ${ }^{10,11}$.

c) En la senectud, siempre debe medirse la PA en posición de pie y debe tenerse presente la posibilidad de pseudohipertensión (falsa elevación de la PA por rigidez arterial que dificulta la compresión de la arteria) ${ }^{12}$.

\section{Diagnóstico de HTA}

Se considera hipertensa a toda mujer $\geq 18$ años con PAS $\geq 140$ y/o PAD $\geq 90 \mathrm{mmHg}$, obtenidas del promedio de 2 o más lecturas de PA en 2 días diferentes ${ }^{13}$. También se puede diagnosticar HTA, a través de la medición de la PA fuera de la consulta médica (valor límite 135/85 mmHg), siguiendo pautas establecidas ${ }^{14}$.

En toda hipertensa, se debe evaluar primero su riesgo cardiovascular $(\mathrm{CV})$ y a continuación descartar HTA secundaria. Una HTA secundaria debe buscarse cuando existen elementos sugerentes de causas secundarias, como edad menor de 30 años, HTA de difícil control y hallazgos característicos al examen físico y, en aquellas con alteraciones de exámenes "iniciales" como creatinina plasmática, electrolitos plasmáticos y orina completa ${ }^{13}$.

\section{Estratificación de la HTA}

Para determinar el riesgo CV es fundamental realizar una estratificación que incluya la magnitud de la HTA, el número de factores de riesgo cardiovascular (FRCV) asociados, la presencia o ausencia de daño de órganos blanco (DOB), y el antecedente de enfermedad CV aterosclerótica clínica (ECVA). Aunque no aparece en las escalas de estratificación, no debe olvidarse la historia familiar de ECVA prematura, que implica mayor riesgo $\mathrm{CV}$.

Recientemente se ha demostrado una asociación entre HTA en el embarazo y desarrollo pos- 
terior de $\mathrm{ECV}^{15-17}$. La preeclampsia (PE) aumenta el riesgo de ECVA en 2 a 3 veces ${ }^{18}$, convirtiéndola en uno de los factores de riesgo más precoces, con un peso semejante al síndrome metabólico $(\mathrm{SM})^{19}$. Esto hace necesario incluir la historia reproductiva (HTA gestacional, mortinatos, prematuros, recién nacidos de bajo peso y proteinuria en el embarazo).

\section{Factores de riesgo cardiovasculares asociados}

La mayoría de las hipertensas presentan otros FRCV asociados, cuyo efecto puede ser aditivo y/o multiplicativo. En el estudio Framingham, menos del $20 \%$ de la mujeres presentaban HTA como único FRCV ${ }^{20}$. La ENS 2003 también observó esta

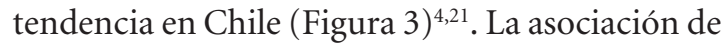
los diferentes FRCV aumenta gradualmente con la magnitud de la obesidad y con el incremento de peso a través del tiempo.

\section{HTA secundaria}

Diferentes estudios han demostrado una prevalencia entre 5 a $10 \%$ en hipertensas referidas ${ }^{22}$. Las causas más frecuentes son: ACO, enfermedad renal crónica (ERC), hiperaldosteronismo primario (HAP) y la HTA renovascular (HTA RV) ${ }^{22,23}$.

Es primordial descartar entidades que pueden complicarse durante el embarazo como nefritis lúpica, HTA RV y feocromocitoma ${ }^{24}$. A diferencia de otras hipertensiones crónicas, que pueden

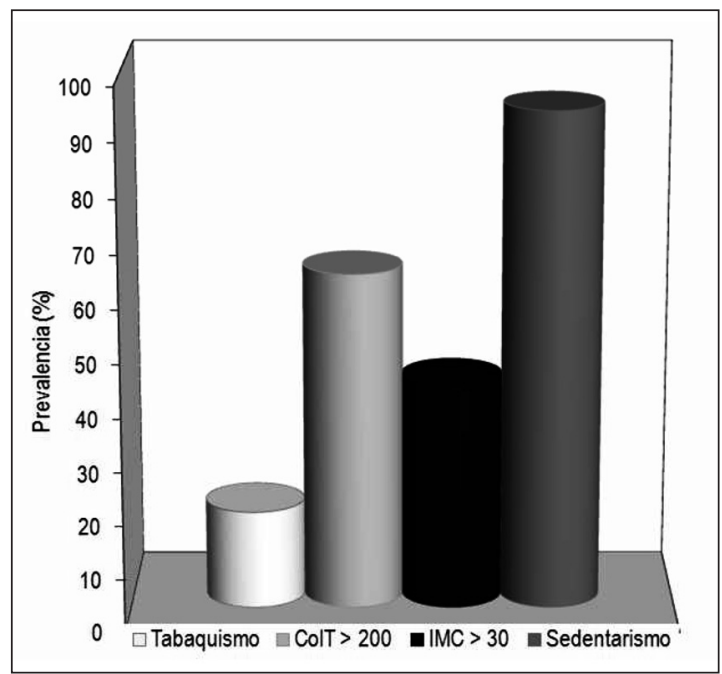

Figura 3. Factores de riesgo CV en hipertensas en la Encuesta Nacional de Salud 2003. mostrar mejoría de la PA durante el segundo trimestre, en el HAP el beneficio se prolonga hasta el tercer trimestre, e incluso hasta el postparto. El descenso se atribuye a la inhibición competitiva de aldosterona por la progesterona placentaria y a la estimulación de los sistemas vasodilatadores en el embarazo ${ }^{25}$. Sin embargo, hay casos de HAP asociados a embarazo en que se agrava la $\mathrm{HTA}^{26}$.

En la premenopausia, la HTA RV se debe generalmente a una displasia fibromuscular, que puede asociarse a hipercoagulabilidad con riesgo de infartos renales. La displasia suele acentuarse en el embarazo, y deja de progresar en la menopausia ${ }^{27}$.

La menopausia "per se" puede causar taquicardia, bochornos, sudoración y/o cambios de carácter, que pueden sugerir el diagnóstico de feocromocitoma, que se caracteriza por HTA asociada a la tríada de cefalea, palpitaciones y sudoración. Sólo se justifica buscar un feocromocitoma en presencia de HTA asociada a esta tríada.

Existen una serie de drogas relacionadas con HTA como anorexígenos, antiinflamatorios no esteroidales, antijaquecosos, antidepresivos y $\mathrm{ACO}^{28,29}$.

\section{Premenopausia}

\section{HTA inducida por ACO}

Los ACO tienden a aumentar la PA en toda mujer, aunque el incremento es leve. Sin embargo, un pequeño porcentaje puede desarrollar HTA e incluso HTA maligna ${ }^{24,30}$. El estudio "Nurses' Health Study" demostró que los ACO conferían un riesgo relativo de HTA de 1,8 en usuarias crónicas, siendo mayor en aquellas que habían utilizado ACO por más de 6 años. Sin embargo, el número de mujeres que desarrollaron HTA fue de 41 casos en 10.000 mujeres en un $\mathrm{año}^{31}$.

Los factores predisponentes más importantes son: antecedente familiar de HTA, historia de síndromes hipertensivos del embarazo (SHE), diabetes mellitus, edad > de 35 años, obesidad y ERC.

Esta HTA depende de la dosis de estrógenos y no de progestágenos. Con las dosis actuales menores a $50 \mu \mathrm{g}$ de etinilestradiol, el desarrollo de HTA es excepcional ${ }^{31,32}$.

Los mecanismos de HTA por ACO no están aclarados. Se considera que pudiese ser un tipo de respuesta idiosincrática ${ }^{24}$, o una activación del sistema renina-angiotensina-aldosterona (SRAA), 
lo que se apoya en la respuesta a inhibidores de la enzima convertidora (IECA). Sin embargo, las pacientes que desarrollan HTA tienen un incremento de angiotensinógeno semejante a las que permanecen normotensas. Se han planteado también efectos de dosis altas de etinilestradiol, como: aumento de actividad de dopamina hidroxilasa, efectos vasculares directos e inducción de resistencia insulínica ${ }^{33}$.

Los progestágenos pueden inducir elevación marginal de PA por su capacidad androgénica, pero en la mayoría predomina el efecto bloqueador del receptor mineralocorticoídeo ${ }^{34}$.

El tratamiento es la discontinuación de los ACO. La PA se normaliza en uno a tres meses. Si no fuese posible la suspensión, deben iniciarse diuréticos o IECA y utilizar ACO con dosis bajas de estrógenos.

Si se sospecha un HAP, deben suspenderse los ACO, ya que afectan los niveles plasmáticos de renina y aldosterona ${ }^{35,36}$.

\section{Síndromes hipertensivos del embarazo (SHE)}

Los SHE, cuya frecuencia oscila entre un 6 y $8 \%$ de todas las gestaciones ${ }^{37}$, pueden clasificarse según el tiempo de aparición, presencia o ausencia de proteinuria y compromiso multisistémico en: HTA crónica, HTA gestacional, PE, Eclampsia y Síndrome de HELLP ${ }^{38}$.

\section{Aspectos fisiopatológicos}

El crecimiento adecuado del feto depende del desarrollo morfológico y funcional de la unidad útero-placentaria. Este se logra por la creación de la red vascular de las vellosidades placentarias, y por la transformación de las arterias uterinas espirales que pierden su conformación tubular y capa muscular, convirtiéndose en vasos saculares de amplio lumen sin reactividad a estímulos vasoactivos. Para bañar de sangre materna las vellosidades placentarias con una fracción creciente del gasto cardíaco, la madre aumenta el débito cardíaco y el volumen plasmático; este último cambio se realiza a expensas de una elevación progresiva de la actividad de renina y de aldosterona en plasma, hasta alcanzar niveles 7 a 8 veces mayores que los basales ${ }^{39}$.

En la HTA no proteinúrica (ie, HTA transitoria o exacerbación de una HTA pregestacional) existiría una capacidad reducida de estimular factores vasodilatadores (ej: prostaciclina, calicreína, y angiotensina ${ }^{1-7}$ ).

La PE en cambio deriva de un defecto en la transformación de las arterias espirales, proceso que se desarrolla durante las primeras 20 semanas del embarazo, y que sólo tiene expresión a nivel de ultrasonografía de las arterias uterinas. En una etapa tardía se expresa el síndrome multisistémico por disfunción endotelial (HTA, aumento de la permeabilidad/edema, proteinuria), y en los casos más graves reducción de la función renal, daño hepático, encefalopatía y coagulación intravascular. Esta disfunción endotelial puede tener un componente pregestacional, y agravarse por la deportación de factores derivados de la placenta isquémica. Por otro lado una gran masa placentaria (embarazos múltiples, mola hidatidiforme) puede provocar disfunción de un endotelio previamente normal (Figura 4).

\section{HTA arterial crónica}

Definida cuando la PA se eleva antes de las 20 semanas de embarazo. En Estados Unidos, la prevalencia de HTA crónica en el embarazo ha aumentado progresivamente, y se estima actualmente en un $3 \%{ }^{40}$. Esta entidad puede complicarse induciendo una PE, sobre todo cuando la función renal está alterada.

En esta HTA, la mujer no debe hacer ejercicio físico ni reducir la ingesta de sal. El reposo en decúbito lateral mejora la perfusión placentaria. Si la PA persiste elevada, con $\mathrm{PAD} \geq 100 \mathrm{mmHg}$, debe iniciarse tratamiento farmacológico para evitar complicaciones $\mathrm{CV}$ en la madre, reconociendo que la reducción de la PA puede disminuir la perfusión placentaria. No se ha demostrado que el tratamiento farmacológico prevenga la aparición de PE.

Los antihipertensivos seguros y eficaces para la paciente y el feto son metildopa y labetalol, existiendo para ambos seguimiento alejado de niños expuestos in utero ${ }^{41}$. En segunda línea se encuentran: hidralazina y nifedipino de liberación prolongada $^{42}$ (Tabla 1$)$.

Los $\beta$-bloqueadores (BB) son antihipertensivos efectivos, pero se ha reportado retardo del crecimiento intrauterino (RCIU) cuando se ha usado atenolol durante el segundo trimestre ${ }^{43}, y$ bradicardia e hipoglicemia neonatal en el tercer trimestre ${ }^{38}$.

Los datos sobre los bloqueadores de los canales del calcio son limitados. Son potentes tocolíticos y pueden producir hipotensión marcada cuando se usan simultáneamente con sulfato de magnesio. El nifedipino, que es el más estudiado, ha demos- 


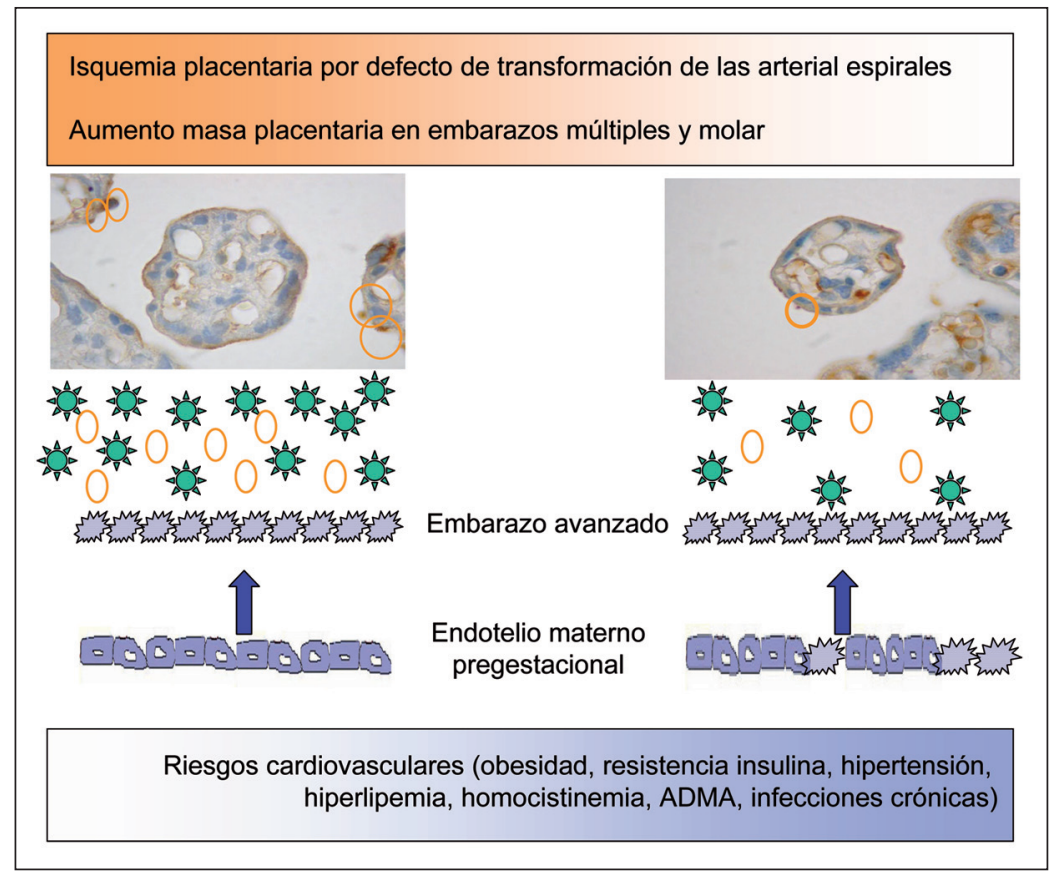

Figura 4. Esquema que muestra la relación entre la placenta y el endotelio materno en condiciones de isquemia o mayor masa placentaria, y de factores de riesgo cardiovascular subyacentes. Las microfotografías representan vellosidades placentarias con yemas de sinciciotrofoblasto (en círculos naranjas) antes de ser deportadas a la circulación materna, junto a una variedad de factores (estrellas verdes: autoanticuerpos agonistas del receptor AT1 R de angiotensina II, dimetilarginina asimétrica, especies reactivas de oxígeno). Tanto las micropartículas de sinciciotrofoblasto como los factores solubles provocan disfunción del endotelio (células con bordes estrellados) previamente sano (células de bordes lisos) o con disfunción.
Tabla 1. Antihipertensivos permitidos en el embarazo

\begin{tabular}{|lc|}
\hline Drogas & Dosis (mg/día) \\
\hline Metildopa & $250-3.000$ en 2 a 4 dosis \\
Labetalol & $200-1.200$ en 2 a 3 dosis \\
\hline Nifedipino & $30-120$ en 1 dosis* \\
Hidralazina & $50-300$ en 2 a 3 dosis \\
Clonidina & $0,1-0,6$ en 2 a 3 dosis \\
Metoprolol & $25-100$ en 1 dosis \\
Hidroclorotiazida & $12,5-25$ en 1 dosis \\
\hline
\end{tabular}

*Liberación prolongada.

trado disminuir la PA y mejorar la función renal, sin afectar el flujo sanguíneo umbilical.

Los diuréticos pueden mantenerse durante el embarazo si se iniciaron antes de la concepción, especialmente en presencia de $\mathrm{ERC}^{43}$. Ya que pueden agravar la depleción de volumen y promover la vasoconstricción reactiva, deben evitarse en la PE.

Los IECA están contraindicados en el embarazo. Afectan el desarrollo renal fetal, causando anuria y oligohidramnios. Las complicaciones reportadas incluyen: hipoplasia pulmonar, deformidades craneofaciales y displasia renal ${ }^{44,45}$.
Los antagonistas del receptor de angiotensina II (ARAII) ejercen un efecto similar a los IECA sobre la circulación renal fetal y pueden, potencialmente, causar malformaciones fetales similares.

\section{Preeclampsia}

Alza de la PA sobre 140/90 mmHg después de las 20 semanas del embarazo, asociada a proteinuria $>300 \mathrm{mg} /$ día y edema. Puede presentarse con cefalea, dolor epigástrico, fotopsias, náuseas y vómitos.

Constituyen condiciones de riesgo para PE: HTA crónica, obesidad, diabetes mellitus, gestaciones múltiples, primigestas en edades extremas y trombofilias. Las complicaciones van desde RCIU hasta eclampsia y síndrome de HELLP, con muerte materna y fetal.

El tratamiento de la PE es la expulsión de la placenta, pero la interrupción del embarazo depende de la viabilidad fetal. Si el feto es maduro se debe interrumpir el embarazo. De lo contrario, debe mantenerse el embarazo y valorar el estado general de la madre y el feto con análisis seriados de sangre y orina, ecografía obstétrica y monitorización fetal, que se repiten según la gravedad. Si la PAD es $>100 \mathrm{~mm} \mathrm{Hg}$, se aconseja usar antihipertensivos. En PE grave, conviene sedar a 
la madre y prevenir las convulsiones con sulfato de magnesio. Durante y después del parto deben continuarse los controles y tratamientos. El riesgo de eclampsia persiste hasta 7 días post parto. En el puerperio debe confirmarse la normalidad de la PA y la desaparición de la proteinuria.

Para prevenir la PE se han intentado varias estrategias, desde la aspirina en dosis bajas hasta la suplementación con calcio, sin demostrar una clara efectividad ${ }^{38,40}$.

Respecto al riesgo de HTA en años sucesivos, las primigrávidas con PE y sin HTA previa ni otros FRCV conocidos, pueden desarrollar HTA con la misma frecuencia que la población general. En cambio, aquellas que experimentan PE en un segundo embarazo tienen un riesgo muy superior de HTA posterior (riesgo relativo entre 3,4 a 8,1) ${ }^{46}$.

La probabilidad de presentar PE en un segundo embarazo es baja, cuando ésta ha sido moderada, de inicio tardío y sin otros FRCV. La PE que aparece antes de la semana 28 se asocia a $65 \%$ de recurrencia en embarazos posteriores. También la PE temprana predice una alta incidencia futura de HTA crónica, de hasta 55\%, y de eventos $\mathrm{CV}^{47,48}$.

\section{Hipertensión gestacional}

Llamada previamente HTA transitoria, se caracteriza por la aparición de HTA no proteinúrica después de las 20 semanas. Estas mujeres tienen un curso benigno, pero pueden desarrollar HTA en futuros embarazos y tienen alto riesgo de HTA crónica $^{38}$.

\section{Post-parto y lactancia}

La HTA de novo, que aparece desde las 4 horas hasta 6 semanas del post-parto $(6 \%)$, puede ser secundaria a PE, tiroiditis post-parto, sobrecarga de volumen por soluciones salinas o medicamentos como metilergonovina (Metergin), oxitocina, $\mathrm{AINE}^{49}$, y bromocriptina, droga supresora de la lactancia ${ }^{50}$.

Tradicionalmente se menciona que la PA de la PE se normaliza a las 6 semanas. Sin embargo un estudio mostró que en $43 \%$ la PA se normalizaba al mes del puerperio, y que $57 \%$ seguía con HTA a los 6 meses $^{51}$. En este último caso deben estudiarse otras etiologías.

La tiroiditis post-parto, de etiología autoinmune, también puede manifestarse con HTA. El diagnóstico se basa en la medición de TSH, T4, T3 y anticuerpos antitiroídeos.

Si hay sospecha de HAP, por pérdida del efecto protector de la progesterona, se sugiere esperar hasta 4 semanas post parto para efectuar el estudio ${ }^{49}$.

El manejo farmacológico debe ser guiado según la etiología y el lactancia. Si la paciente está amamantando, debe considerarse la excreción del antihipertensivo por la leche materna y sus potenciales efectos en el recién nacido (Tabla 2).

En relación a los antihipertensivos recomendados para este período debe mencionarse ${ }^{52}$ :

a) Se prefieren los antihipertensivos tradicionales, metildopa e hidralazina.

b) Los diuréticos tiazídicos y de asa afectan la lactancia, y pueden inducir diuresis e hipovolemia en el recién nacido.

c) Diuréticos retenedores de potasio, así como espironolactona, pueden usarse.

d) Entre los alfa-beta bloqueadores, labetalol es muy seguro.

e) Los bloqueadores del SRA no se prefieren porque la madurez renal se alcanza a los 2 años.

f) Existen insuficientes datos para el uso de bloqueadores de los canales de calcio.

\section{Postmenopausia}

\section{Hipertensión postmenopáusica}

La hipertensión postmenopáusica (HTAPM) podría considerarse como una forma de HTA secundaria. Aunque los resultados de estudios

Tabla 2. Excreción de antihipertensivos en la leche materna

\begin{tabular}{|lc|}
\hline Droga & Razón leche/plasma \\
\hline Atenolol & 3,0 \\
\hline Clonidina & 1,5 \\
\hline Diltiazem & 1,0 \\
\hline Propanolol & 0,6 \\
\hline Hidralazina & 0,5 \\
\hline Hidroclorotiazida & 0,4 \\
Verapamilo & 0,4 \\
\hline Metildopa & 0,3 \\
\hline Captopril & 0,1 \\
\hline Enalapril & 0,01 \\
\hline
\end{tabular}


longitudinales y transversales sobre el rol de la menopausia en la patogénesis de la HTA son discordantes, está claro que su prevalencia aumenta después de la menopausia ${ }^{1}$.

\section{Aspectos epidemiológicos}

La PAS en la mujer premenopáusica es menor que la del hombre, pero sube y casi se iguala a la de éste en la postmenopausia. La PAD, tanto en la pre como postmenopausia, es siempre menor en la mujer. Esto hace que la presión de pulso de la mujer sea mayor después de los 50 años ${ }^{53}$.

El aumento de la PA en la postmenopausia es independiente del índice de masa corporal (IMC) $y$ de la edad ${ }^{54}$. Hasta el momento no se ha descrito una relación entre este tipo de HTA y el antecedente de SHE. Tampoco se conoce relación entre el uso prolongado de ACO y desarrollo de $\mathrm{HTAPM}^{55}$. Estudios epidemiológicos han demostrado que en la menopausia aumenta la prevalencia de otros factores de riesgo hipertensivo, como sedentarismo, obesidad y SM, que asociados a la perdida de los estrógenos y al envejecimiento vascular, elevarían la $\mathrm{PA}^{55}$.

\section{Aspectos fisiopatológicos}

Los mecanismos responsables del aumento de la PA en mujeres postmenopáusicas son complejos y multifacéticos. Los estrógenos participan en muchas vías que regulan la PA como aumento de la biodisponibilidad del óxido nítrico endotelial, inhibición del SRAA y del sistema de las endotelinas, disminución de la actividad del sistema nervioso autonómico simpático, e inhibición de la proliferación de músculo liso vascular. A nivel renal, disminuyen la reabsorción tubular de sodio al inhibir el SRAA intrarrenal y aumentar la acción del óxido nítrico. Las mujeres sometidas a histerectomía y ooforectomia bilateral se hacen sensibles a la sal ${ }^{56}$, que les conferiría mayor riesgo $\mathrm{CV}$, independiente de la PA y de los FRCV tradicionales ${ }^{57}$.

\section{Terapia de reemplazo hormonal}

No puede atribuirse un rol causal de la HTA$\mathrm{PM}$ a la terapia de reemplazo hormonal (TRH), ya que la probabilidad de aumentar la PA con la TRH en las hipertensas es muy baja ${ }^{55}$. Más aún, aquellas mujeres que reciben TRH presentan mayor descenso nocturno de la PA que las no tratadas ${ }^{24}$. Aunque la pérdida de los estrógenos participe en la patogenia de la HTAPM, no se recomienda la TRH para su prevención ni tratamiento ${ }^{58}$.

\section{Tratamiento no farmacológico}

Debe ser instaurado en toda mujer hipertensa, ya sea como tratamiento inicial (con riesgo CV bajo-moderado), o complementando el tratamiento farmacológico ${ }^{59}$.

Las medidas no farmacológicas más eficaces se señalan en la Tabla $3^{60}$.

Respecto a la reducción de peso, no todas las mujeres con sobrepeso presentan una respuesta similar en el descenso de la PA con dietas bajas en calorías. Este depende de la magnitud en la baja de peso y de la edad ${ }^{61}$. Las mujeres menores de 55 años suelen ser sensibles a la restricción calórica, lo que no sucede tan claramente en las mayores de 60 años $^{62}$. En un meta-análisis, por cada kilo de peso perdido se produce un descenso de 1,6 y 1,3 mmHg para PAS y PAD respectivamente ${ }^{63}$. Dos fenómenos son relevantes frente a la reducción de peso: a) las dietas hipocalóricas en postmenopáusicas pueden reducir la densidad ósea, y b) las mujeres que posteriormente recuperan peso, presentan mayor elevación de la PA y cambios en la distribución de la grasa corporal desde un tipo ginecoide a androide ${ }^{61}$.

Las postmenopáusicas son más sensibles al sodio que las premenopáusicas, y su reducción logra un mayor descenso de PA. La reducción de la ingesta de sodio produce camvbios mas marcados para PAS, dependientes de la cantidad de sal y de la edad. Sin embargo, sólo $20 \%$ se mantiene en el consumo deseado de sodio de $75 \mathrm{mEq} /$ día, equivalente a 4,4 gramos de sal ${ }^{64}$.

\section{Tabla 3. Medidas no farmacológicas eficaces en el descenso de la PA}

Reducción de peso
Reducción del consumo de sal sódica
Reducción del consumo de alcohol
Aumento de la actividad física
Aumento del consumo de frutas y verduras
Disminución del consumo de grasa total y grasa saturada
Abandono del tabaco
Adopción de una dieta mediterránea


El ejercicio aeróbico regular en mujeres, produce una reducción de $2,3 \mathrm{mmHg}$ en la PAS y $1,2 \mathrm{mmHg}$ en la $\mathrm{PAD}^{65}$ independiente del peso corporal o dieta.

La ingesta de ácidos grasos insaturados, como aceite de oliva, produce un descenso significativo de la PA en hipertensas ${ }^{61}$.

Los suplementos orales de calcio, también pueden reducir la PA, aunque no en todas las mujeres $^{59}$.

En cuanto al café, se recomienda no más de 4 tazas al día.

La reducción del consumo de alcohol a un trago al día ${ }^{66}$ se asocia a una reducción de la PA dosis dependiente, similar entre hipertensas o no hipertensas.

El aumento en la ingesta de potasio reduce la $\mathrm{PA}$, pero su efecto es dependiente de la magnitud de la ingesta de sodio. Actualmente, se recomienda una ingesta de potasio de $120 \mathrm{mEq} /$ día (4,7 gr/día), como la aportada por la dieta del estudio "Dietary Approaches to Stop Hypertension" (DASH) ${ }^{67}$.

Estudio DASH, demostró que una dieta rica en frutas, vegetales, lácteos bajos en grasas, fibra dietética, potasio, calcio y magnesio, y pobre en grasas totales, grasas saturadas y colesterol, disminuía la PA de manera independiente de la reducción de peso y del sodio ${ }^{68}$. En hipertensas, la PAS y PAD disminuyeron en 11,6 y 5,3 mmHg, respectivamente. Esta dieta, de mayor efectividad que otras, ha sido adoptada por el VII reporte del JNC y por la $\mathrm{AHA}^{66}$ para la prevención y tratamiento de la HTA. Además, en el estudio "Reduced Dietary Sodium and the Dietary Approaches to Stop Hypertension" (DASH-sodium) se probó el efecto de la dieta DASH con y sin restricción de $\mathrm{Na}$ (50 vs $150 \mathrm{mEq} /$ día) versus dieta control con y sin restricción de $\mathrm{Na}$, demostrándose un efecto aditivo de ambos componentes, especialmente en mujeres ${ }^{69}$.

\section{Tratamiento farmacológico}

Los primeros estudios controlados en hipertensión leve que incluyeron a mujeres, como el Hypertension Detection and Follow-up Program $(\mathrm{HDFP})^{70}$ y el Medical Research Council (MRC) $)^{71}$, sugirieron que aquellas con HTA leve a moderada se beneficiaban menos que los hombres, y que más aún las mujeres menores de 55 años podían perjudicarse $^{72}$. En los noventa, los primeros metanálisis con más de 20.000 mujeres, mostraron una reducción de $38 \%$ de los ACV, pero sólo un 12\% de reducción en los eventos coronarios $(p=0,059)^{72,73}$, beneficios significativamente menores que en los hombres ${ }^{73}$. Sin embargo, un metanálisis reciente, con 87.349 mujeres, ha permitido demostrar beneficios similares a los observados en los hombres, incluso para la prevención de $\mathrm{CC}^{74}$.

La respuesta hipotensora de los diferentes antihipertensivos es similar entre hombres y muje$\mathrm{res}^{74}$, y no parece existir una diferencia de impacto clínico según la familia de antihipertensivos en la reducción de los eventos CV por género. Una excepción a esto, fue el estudio LIFE (Losartan Intervention For Endpoint reduction in hypertension $s t u d y$ ), en el que las hipertensas con hipertrofia ventricular se beneficiaron más con losartan que con atenolol en la prevención de $\mathrm{ACV}^{75,76}$.

En las mujeres postmenopáusicas, los BB tradicionales no debiesen ser de primera línea, por la alta prevalencia de SM y porque dificultan el descenso de peso. Éstas pudiesen beneficiarse más de las tiazidas, por su sensibilidad a la sal y porque reducen las fracturas óseas (77). Así mismo, considerando el incremento de la actividad del SRAA, los IECA y los ARAII pueden también ser particularmente apropiados, especialmente dado que una hipertensa tiene el doble de riesgo de desarrollar diabetes respecto a una normotensa ${ }^{78}$.

Finalmente, los efectos colaterales de los antihipertensivos son más frecuentes en la mujer que en el hombre, como hiponatremia e hipokalemia secundaria a tiazidas ${ }^{24}$, edema por antagonistas del calcio, y tos inducida por los IECA, que es tres veces más frecuente ${ }^{24}$.

\section{Referencias}

1. Hajjar I, Kotchen TA. Trends in prevalence, awareness, treatment, and control of hypertension in the United States, 1988-2000. JAMA 2003; 290 (2): 199-206.

2. Kannel WB, Dawber TR, Kagan A, Revotskie N, Stokes J, $3 \mathrm{rd}$. Factors of risk in the development of coronary heart disease-six year follow-up experience. The Framingham Study. Ann Intern Med 1961; 55: 33-50.

3. Levy D, Larson MG, Vasan RS, Kannel WB, Ho KK. The progression from hypertension to congestive heart failure. JAMA 1996; 275 (20): 1557-62.

4. www.minsal.cl.

5. Wassertheil-Smoller S, Anderson G, Psaty BM, Black HR, Manson J, Wong N, et al. Hypertension and its 
treatment in postmenopausal women: baseline data from the Women's Health Initiative. Hypertension 2000; 36 (5): 780-9.

6. Pickering TG, Hall JE, Appel LJ, Falkner BE, Graves JW, Hill MN, et al. Recommendations for blood pressure measurement in humans: an AHA scientific statement from the Council on High Blood Pressure Research Professional and Public Education Subcommittee. J Clin Hypertens (Greenwich) 2005; 7 (2): 102-9.

7. Stolt M, Sjonell G, Astrom H, Rossner S, Hansson L. Improved accuracy of indirect blood pressure measurement in patients with obese arms. Am J Hypertens. 1993; 6 (1): 66-71.

8. Perloff D, Grim C, Flack J, Frohlich ED, Hill M, McDonald $\mathrm{M}$, et al. Human blood pressure determination by sphygmomanometry. Circulation 1993; 88 (5 Pt 1): 2460-70.

9. Parati G, Stergiou GS, Asmar R, Bilo G, de Leeuw P, Imai $Y$, et al. European Society of Hypertension guidelines for blood pressure monitoring at home: a summary report of the Second International Consensus Conference on Home Blood Pressure Monitoring. J Hypertens 2008; 26 (8): 1505-26.

10. Little P, Barnett J, Barnsley L, Marjoram J, FitzgeraldBarron A, Mant D. Comparison of agreement between different measures of blood pressure in primary care and daytime ambulatory blood pressure. BMJ. 2002; 325 (7358): 254.

11. Pickering TG, Miller NH, Ogedegbe G, Krakoff LR, Artinian NT, Goff D. Call to action on use and reimbursement for home blood pressure monitoring: Executive Summary. A joint scientific statement from the American Heart Association, American Society of Hypertension, and Preventive Cardiovascular Nurses Association. J Clin Hypertens (Greenwich) 2008; 10 (6): 467-76.

12. Wright JC, Looney SW. Prevalence of positive Osler's manoeuver in 3387 persons screened for the Systolic Hypertension in the Elderly Program (SHEP). J Hum Hypertens 1997; 11 (5): 285-9.

13. Chobanian AV, Bakris GL, Black HR, Cushman WC, Green LA, Izzo JL Jr, et al. The Seventh Report of the Joint National Committee on Prevention, Detection, Evaluation, and Treatment of High Blood Pressure: the JNC 7 report. JAMA 2003; 289 (19): 2560-72.

14. Campbell NR, Khan NA, Hill MD, Tremblay G, Lebel M, Kaczorowski J, et al. 2009 Canadian Hypertension Education Program recommendations: the scientific summary-an annual update. Can J Cardiol 2009; 25 (5): 271-7.

15. Germain AM, Romanik MC, Guerra I, Solari S, Reyes
MS, Johnson RJ, et al. Endothelial dysfunction: a link among preeclampsia, recurrent pregnancy loss, and future cardiovascular events? Hypertension 2007; 49 (1): 90-5.

16. Bellamy L, Casas JP, Hingorani AD, Williams DJ. Preeclampsia and risk of cardiovascular disease and cancer in later life: systematic review and meta-analysis. BMJ 2007; 335 (7627): 974.

17. Carty DM, Delles C, Dominiczak AF. Preeclampsia and future maternal health. J Hypertens 2010; 28 (7): 134955.

18. Roberts JM, Hubel CA. Pregnancy: a screening test for later life cardiovascular disease. Womens Health Issues 2010; 20 (5): 304-7.

19. Lloyd-Jones D, Adams RJ, Brown TM, Carnethon M, Dai S, De Simone G, et al. Heart disease and stroke statistics-2010 update: a report from the American Heart Association. Circulation 2010; 121 (7): e46-e215.

20. Wilson PW, Kannel WB, Silbershatz H, D’Agostino RB. Clustering of metabolic factors and coronary heart disease. Arch Intern Med 1999; 159 (10): 1104-9.

21. Margozzini P, Rigotti A, Ferreccio C, Quezada N, Garrido M, Valdes G. Hypertension and the cardiometabolic syndrome in Chile: a review of concepts and consequences for the developing world. Ther Adv Cardiovasc Dis 2007; 1 (1): 83-90.

22. Omura M, Saito J, Yamaguchi K, Kakuta Y, Nishikawa T. Prospective study on the prevalence of secondary hypertension among hypertensive patients visiting a general outpatient clinic in Japan. Hypertens Res 2004; 27 (3): 193-202.

23. Sinclair AM, Isles CG, Brown I, Cameron H, Murray GD, Robertson JW. Secondary hypertension in a blood pressure clinic. Arch Intern Med 1987; 147 (7): 1289-93.

24. August P, Oparil S. Hypertension in women. J Clin Endocrinol Metab 1999; 84 (6): 1862-6.

25. Germain AM, Kottman C, Valdes G. [Primary aldosteronism and pregnancy: report of 2 cases]. Rev Med Chile 2002; 130 (12): 1399-405.

26. Okawa T, Asano K, Hashimoto T, Fujimori K, Yanagida K, Sato A. Diagnosis and management of primary aldosteronism in pregnancy: case report and review of the literature. Am J Perinatol 2002; 19 (1): 31-6.

27. Olin JW. Recognizing and managing fibromuscular dysplasia. Cleve Clin J Med 2007; 74 (4): 273-4, 7-82.

28. Siebenhofer A, Horvath K, Jeitler K, Berghold A, Stich AK, Matyas E, et al. Long-term effects of weight-reducing drugs in hypertensive patients. Cochrane Database Syst Rev 2009 (3): CD007654.

29. Woods JW. Oral contraceptives and hypertension. Lancet 1967; 2 (7517): 653. 
30. Petitti DB, Klatsky AL. Malignant hypertension in women aged 15 to 44 years and its relation to cigarette smoking and oral contraceptives. Am J Cardiol 1983; 52 (3): 297-8.

31. Chasan-Taber L, Willett WC, Manson JE, Spiegelman D, Hunter DJ, Curhan G, et al. Prospective study of oral contraceptives and hypertension among women in the United States. Circulation 1996; 94 (3): 483-9.

32. Fasce E, Ibanez P, Nieto C. [A controlled study of the effect of oral contraceptives on blood pressure]. Rev Med Chile 1995; 123 (2): 158-64.

33. Boldo A, White WB. Blood pressure effects of the oral contraceptive and postmenopausal hormone therapies. Endocrinol Metab Clin North Am 2011; 40 (2): 419-32, ix.

34. ACOG practice bulletin. No. 73: Use of hormonal contraception in women with coexisting medical conditions. Obstet Gynecol 2006; 107 (6): 1453-72.

35. Pizzolo F, Pavan C, Corrocher R, Olivieri O. Laboratory diagnosis of primary aldosteronism, and drospirenoneethinylestradiol therapy. Am J Hypertens 2007; 20 (12): 1334-7.

36. Ahmed AH, Gordon RD, Taylor PJ, Ward G, Pimenta E, Stowasser M. Effect of contraceptives on aldosterone/ renin ratio may vary according to the components of contraceptive, renin assay method, and possibly route of administration. J Clin Endocrinol Metab 2011; 96 (6): 1797-804.

37. Report of the National High Blood Pressure Education Program Working Group on High Blood Pressure in Pregnancy. Am J Obstet Gynecol 2000; 183 (1): S1-S22.

38. Garovic VD. Hypertension in pregnancy: diagnosis and treatment. Mayo Clin Proc 2000; 75 (10): 1071-6.

39. Valdes G, Corthorn J. Review: The angiogenic and vasodilatory utero-placental network. Placenta 2011; 32 Suppl 2: S170-5.

40. Seely EW, Ecker J. Clinical practice. Chronic hypertension in pregnancy. N Engl J Med 2011; 365 (5): 439-46.

41. Chan WS, Koren G, Barrera M, Rezvani M, KnittelKeren D, Nulman I. Neurocognitive development of children following in-utero exposure to labetalol for maternal hypertension: a cohort study using a prospectively collected database. Hypertens Pregnancy 2010; 29 (3): 271-83.

42. Podymow T, August P. Antihypertensive drugs in pregnancy. Semin Nephrol 2011; 31 (1): 70-85.

43. ACOG Practice Bulletin. Chronic hypertension in pregnancy. ACOG Committee on Practice Bulletins. Obstet Gynecol 2001; 98 (1): suppl 177-85.

44. Valdes G, Marinovic D, Falcon C, Chuaqui R, Duarte I. Placental alterations, intrauterine growth retardation and teratogenicity associated with enalapril use in pregnant rats. Biol Neonate 1992; 61 (2): 124-30.

45. Leeman L, Fontaine P. Hypertensive disorders of pregnancy. Am Fam Physician 2008; 78 (1): 93-100.

46. Harskamp RE, Zeeman GG. Preeclampsia: At Risk for Remote Cardiovascular Disease. Am J Med Sci 2007; 334 (4): 291-5 10.1097/MAJ.0b013e3180a6f094.

47. Valdes G, Quezada F, Marchant E, von Schultzendorff A, Moran S, Padilla O, et al. Association of remote hypertension in pregnancy with coronary artery disease: a case-control study. Hypertension 2009; 53 (4): 733-8.

48. Williams D. Long-term complications of preeclampsia. Semin Nephrol 2011; 31 (1): 111-22.

49. Ghuman N, Rheiner J, Tendler BE, White WB. Hypertension in the postpartum woman: clinical update for the hypertension specialist. J Clin Hypertens (Greenwich) 2009; 11 (12): 726-33.

50. Watson DL, Bhatia RK, Norman GS, Brindley BA, Sokol RJ. Bromocriptine mesylate for lactation suppression: a risk for postpartum hypertension? Obstet Gynecol 1989; 74 (4): 573-6.

51. Podymow T, August P. Hypertension in pregnancy. Adv Chronic Kidney Dis 2007; 14 (2): 178-90.

52. Ghanem FA, Movahed A. Use of antihypertensive drugs during pregnancy and lactation. Cardiovasc Ther 2008; 26 (1): 38-49.

53. Safar ME, Smulyan H. Hypertension in women. Am J Hypertens 2004; 17 (1): 82-7.

54. Zanchetti A, Facchetti R, Cesana GC, Modena MG, Pirrelli A, Sega R. Menopause-related blood pressure increase and its relationship to age and body mass index: the SIMONA epidemiological study. J Hypertens 2005; 23 (12): 2269-76.

55. Barton M, Meyer MR. Postmenopausal hypertension: mechanisms and therapy. Hypertension 2009; 54 (1): 11-8.

56. Schulman IH, Aranda P, Raij L, Veronesi M, Aranda FJ, Martin R. Surgical menopause increases salt sensitivity of blood pressure. Hypertension 2006; 47 (6): 1168-74.

57. Morimoto A, Uzu T, Fujii T, Nishimura M, Kuroda S, Nakamura S, et al. Sodium sensitivity and cardiovascular events in patients with essential hypertension. Lancet 1997; 350 (9093): 1734-7.

58. Collins P, Rosano G, Casey C, Daly C, Gambacciani M, Hadji P, et al. Management of cardiovascular risk in the peri-menopausal woman: a consensus statement of European cardiologists and gynaecologists. Eur Heart J 2007; 28 (16): 2028-40.

59. Appel LJ, Giles TD, Black HR, Izzo JL, Jr., Materson BJ, Oparil S, et al. ASH Position Paper: Dietary approaches 
to lower blood pressure. J Clin Hypertens (Greenwich) 2009; 11 (7): 358-68.

60. Williams B, Poulter NR, Brown MJ, Davis M, McInnes GT, Potter JF, et al. Guidelines for management of hypertension: report of the fourth working party of the British Hypertension Society, 2004-BHS IV. J Hum Hypertens 2004; 18 (3): 139-85.

61. Costa FV. Non-pharmacological treatment of hypertension in women. J Hypertens Suppl 2002; 20 (2): S57-61.

62. Kawamura M, Adachi T, Nakajima J, Fujiwara T, Hiramori K. Factors that affect calorie-sensitive and calorie-insensitive reduction in blood pressure during short-term calorie restriction in overweight hypertensive women. Hypertension 1996; 27 (3 Pt 1): 408-13.

63. Staessen J, Fagard R, Lijnen P, Amery A. Body weight, sodium intake and blood pressure. J Hypertens Suppl 1989; 7 (1): S19-23.

64. Midgley JP, Matthew AG, Greenwood CM, Logan AG. Effect of reduced dietary sodium on blood pressure: a meta-analysis of randomized controlled trials. JAMA 1996; 275 (20): 1590-7.

65. Kelley GA. Aerobic exercise and resting blood pressure among women: a meta-analysis. Prev Med 1999; 28 (3): 264-75.

66. Appel LJ, Brands MW, Daniels SR, Karanja N, Elmer PJ, Sacks FM. Dietary approaches to prevent and treat hypertension: a scientific statement from the American Heart Association. Hypertension 2006; 47 (2): 296-308.

67. Appel LJ, Moore TJ, Obarzanek E, Vollmer WM, Svetkey LP, Sacks FM, et al. A Clinical Trial of the Effects of Dietary Patterns on Blood Pressure. New England Journal of Medicine 1997; 336 (16): 1117-24.

68. Appel LJ, Moore TJ, Obarzanek E, Vollmer WM, Svetkey LP, Sacks FM, et al. A clinical trial of the effects of dietary patterns on blood pressure. DASH Collaborative Research Group. N Engl J Med 1997; 336 (16): 1117-24.

69. Sacks FM, Svetkey LP, Vollmer WM, Appel LJ, Bray GA, Harsha D, et al. Effects on blood pressure of reduced dietary sodium and the Dietary Approaches to Stop Hypertension (DASH) diet. DASH-Sodium Collaborative
Research Group. N Engl J Med 2001; 344 (1): 3-10.

70. Five-year findings of the hypertension detection and follow-up program. I. Reduction in mortality of persons with high blood pressure, including mild hypertension. Hypertension Detection and Follow-up Program Cooperative Group. JAMA 1979; 242 (23): 2562-71.

71. MRC trial of treatment of mild hypertension: principal results. Medical Research Council Working Party. Br Med J (Clin Res Ed) 1985; 291(6488): 97-104.

72. Jones CA, Nagpal S. An update: women, hypertension and therapeutic efficacy. Can J Cardiol 2001; 17 (12): 1283-9.

73. Gueyffier F, Boutitie F, Boissel JP, Pocock S, Coope J, Cutler J, et al. Effect of antihypertensive drug treatment on cardiovascular outcomes in women and men. A meta-analysis of individual patient data from randomized, controlled trials. The INDANA Investigators. Ann Intern Med 1997; 126 (10): 761-7.

74. Turnbull F, Woodward M, Neal B, Barzi F, Ninomiya $\mathrm{T}$, Chalmers J, et al. Do men and women respond differently to blood pressure-lowering treatment? Results of prospectively designed overviews of randomized trials. Eur Heart J 2008; 29 (21): 2669-80.

75. Dahlof B, Devereux RB, Kjeldsen SE, Julius S, Beevers G, de Faire U, et al. Cardiovascular morbidity and mortality in the Losartan Intervention For Endpoint reduction in hypertension study (LIFE): a randomised trial against atenolol. Lancet 2002; 359 (9311): 995-1003.

76. Os I, Franco V, Kjeldsen SE, Manhem K, Devereux RB, Gerdts E, et al. Effects of losartan in women with hypertension and left ventricular hypertrophy: results from the Losartan Intervention for Endpoint Reduction in Hypertension Study. Hypertension 2008; 51 (4): 1103-8.

77. Schoofs MW, van der Klift M, Hofman A, de Laet CE, Herings RM, Stijnen T, et al. Thiazide diuretics and the risk for hip fracture. Ann Intern Med 2003; 139 (6): 47682.

78. Andraws R, Brown DL. Effect of inhibition of the reninangiotensin system on development of type 2 diabetes mellitus (meta-analysis of randomized trials). Am J Cardiol 2007; 99 (7): 1006-12. 